\title{
S4 File
}

\section{Additional results experiment 3}

\section{Mimicry effect, response facilitation and inhibition including the factor Happy (SAMT with vignettes)}

\section{Mimicry effect}

We performed a with a repeated measurement ANOVA with factors GROUP (In-group, Outgroup) and THREAT (Happy, Angry/No Personal Threat, Angry/Personal Threat) on the mean difference scores (i.e., RT on incongruent minus congruent trials). This revealed a trend significant GROUP X THREAT interaction $(\mathrm{F}(2,114)=4.386, \mathrm{p}=.015$, partial $\eta 2=.071)$ in the absence of significant main effects (all ps $\leq .26$ ). We carried out planned pairwise comparisons which showed a significantly stronger mimicry effect when Out-group / Personal Threat stimuli were presented than In-group Personal Threat stimuli $(\mathrm{t}(57)=22.76$, $\mathrm{p}=.008$, see Table 1 for details). Moreover, the effect of type of threat was different for the In-group, as shown by a significant difference when In-group / No Personal Threat was compared to In-group / Personal Threat, with a higher mimicry effect for In-group / No Personal Threat $(\mathrm{t}(57)=3.34, \mathrm{p}=.001$, see Table 1 for details $)$. Also the mimicry effect for In-group Happy was higher than for In-group Threat $(\mathrm{t}(57)=2.023, \mathrm{p}=0.048)$. No other significant differences were found (all ps $\geq .303$ ).

We performed a three-way repeated measures ANOVA with the factors THREAT (Happy, Angry/No Personal Threat, Angry/ Personal Threat), GRouP (In-, Out-Group) and CONGRUENCY (Congruent, Incongruent). This revealed a main effect for CONGRUENCY $(\mathrm{F}(1,57)=400.522, \mathrm{p} \leq .001$, partial $\eta 2=.875)$ with faster mean reaction times for congruent compared to incongruent trials (congruent: $\mathrm{M}=476.243, \mathrm{SE}=8.04$; incongruent: $\mathrm{M}=$ 546.655, SE = 9.702). 


\section{Response facilitation}

We performed repeated measures ANOVAs separate on congruent trials with the factors THREAT (Happy, Angry/No Personal Threat, Angry/ Personal Threat), GrouP (In-, OutGroup). This revealed a trend significant GROUP X THREAT interaction $(\mathrm{F}(1,114)=3.008, \mathrm{p}=$ .053 , partial $\eta 2=.05$ ), in the absence of significant main effects (all $\mathrm{ps} \geq .43$ ). Planned comparisons revealed no further significant differences when adding the presentation of happy faces to analyses (all $p$ 's $\geq .163$ ).

\section{Response inhibition}

We performed repeated measures ANOVAs separate on incongruent trials with the factors ThreAt (Happy, Angry/No Personal Threat, Angry/ Personal Threat), GrouP (In-, OutGroup). This revealed no significant main effects for GROUP $(F(1,57)=.49, \mathrm{p}=.487$, partial $\eta 2=.009)$ and a trend significant main effect for THREAT $(\mathrm{F}(1,114)=2.823, \mathrm{p}=.064$, partial $\eta 2=.047)$. No significant effect was found for the interaction GROUP $x$ THREAT $(F(1,114)=$ $.226, p=.798$, partial $\eta 2=.004)$. 